by a combination of cholecystectomy, T-tube drainage of the common bile-duct, and irrigation of the ducts with a solution of oxytetracycline. In view of our complete failure to eradicate E. coli infections by a similar technique the method has not been employed.

The factors which militate against eradication of the infection are probably those also operative in $E$. coli infections. In the early lesion the parenchyma of the affected segment is so grossly disrupted by chronic inflammatory tissue that it is doubtful if bile is secreted into the dilated ducts. Antibiotics excreted in the bile will therefore be ineffective. Further, the replacing tissue in the quiescent phase is relatively avascular and penetration to the foci of infection therein will be impeded. Once stones form in the biliary tree another factor in the maintenance of the infection is added. Whipple (1929) claimed that "the presence of stones, porous and pervious to typhoid bacilli ... reinfects the fresh incoming bile." Tynes and Utz (1962), in a series of elegant experiments, have obtained evidence that this may well be the case.

\section{Summary}

The typhoid-carrier state among the Chinese in Hong Kong differs from that reported in Europe and the U.S.A. in that there is an equal sex incidence and cholecystectomy fails to eradicate the infection. Evidence is presented which is consistent with the carrier state being maintained by a focus of infection within the liver, a focus which is indistinguishable from the early lesion encountered in pyogenic cholangitis due to $E$. coli, the common disease of the biliary tract in Hong Kong. It is concluded that the absence of the gall-bladder carrier may be attributable to the rarity of chronic cholecystitis.

The factors responsible for the persistence of the salmonellae within the liver have not been determined. In view of a significantly higher incidence in typhoid carriers of infection of the bile-ducts with a liver fluke, $C$. sinensis, it is concluded that this infection may be a factor, though it cannot be the only one.
While the majority of carriers remain symptomless excreters, in a minority the infection extends, and the syndrome of recurrent pyogenic cholangitis which develops can be distinguished from that due to $E$. coli only by bacteriological investigation. It would appear that approximately $5 \%$ of cases of recurrent pyogenic cholangitis are due to salmonellae.

Sulphonamides and a variety of antibiotics have been used in an attempt to eradicate the infection, but to no avail. A six-months course of ampicillin and probenecid similarly failed to eradicate the infection in symptomless carriers, symptomless cholecystectomized carriers, and carriers with recurrent pyogenic cholangitis who had been treated surgically.

The public health implications of the reported findings are obvious. These are not restricted to Hong Kong. For example, there are some thousands of Chinese, recent emigrants from Hong Kong, employed in restaurants in the United Kingdom.

\section{REFERENCES}

Ames, W. R., and Robins, M. (1943). Amer. f. publ. Hlth, 33, 221. Aschoff, L. (1924). Lectures on Pathology. Hoeber, New York

Browning, C. H. (1933). Spec. Rep. Ser. med. Res. Coun. (Lond.), No. 179 .

Bullock, W. E. (1963). Amer. F. med. Sci., 246, 42.

Christie, A. B. (1964). Postgrad. med. F., Suppl. to vol. 40, p. 84 Cook, J., Hou, P. C., Ho, H. C., and McFadzean, A J. S. (1954). Brit f. Surg., 42, 188.

Dehler, H. (1907). Münch. med. Wschr., 54, 779.

Digby, K. H. (1930). Brit. F. Surg., 17, 578.

Erlik, D., and Reitler, R. (1960). Lancet, 1, 1216.

Hou, P. C. (1955). F. Path. Bact., 70, 53.

Humbert, R. (1959). Dtsch. med.'Wschr., 84, 742

Loewenthal, H., and Corfield, W. F (1943), 742.

Loewenthal, H., and Corfield, W. F. (1943). Brit. med. f., 2, 105 Fadzean, A. J. S., and Yeung, R. T. T. (1965). Trans. roy. Soc. trop. Med. Hyg., 59, 179

Ong, G. B. (1962). Arch. Surg., 84, 199.

Parker, R. T., Fitzgerald, J. C., Perry, H. D., and Woodward, T. B (1953). भ. clin. Invest., 32, 593.

Rains, A. J. H. (1962). Brit. med. 7., 2, 685

Teoh, T. B. (1963). 7. Path. Bact 86, i23

Trafford, J. A. P., MacLaren, D. M., Lillicrap, D. A., Barnes, R. D. S. Houston, J. C., and Knox, R. (1962). Lancet, 1, 987.

Tynes, B. S., and Utz, J. P. (1962). Ann. intern. Med., 57, 871.

Vogelsang, T. M. (1929). Med. Rev. (Bergen), 46, 1.

Whipple, A. O. (1929). Ann. Surg., 90, 631.

Wilson, G. S., and Miles, A. A. (1964). Topley and Wilson's Principles of Bacteriology and Immunity, 5th ed. Arnold, London.

\title{
Placental Localization by Thermography
}

\author{
K. G. MILLAR,* M.B., CH.B.
}

Brit. med. F., 1966, 1, 1571-1574

An accurate method of locating the placental site without exposing the mother and foetus to irradiation would be a major advance in obstetrics. Thermography-the pictorial representation of body-heat patterns-has been claimed to be such a method (Young, 1964 ; Birnbaum and Kliot, 1965). The present study was undertaken to investigate this application of thermography.

\section{Method}

Theory.-Thermography depicts superficial heat patterns and has been used to investigate breast neoplasms (Lawson, 1956 ; Lloyd Williams, K., Lloyd Williams, F., and Handley, 1960, 1961), vascular occlusions (Barnes, 1963), rheumatoid arthritis, and burns. Placental localization by this method is based on the premise, as yet unproved, that the placenta, being

* Senior House Officer, the Queen Mother's Hospital, Glasgow. an arteriovenous "fistula," has a temperature higher than its immediate environment, and that the heat from this area is transmitted through the adjacent anterior abdominal wall to produce a characteristic pattern of heat emission on its surface.

Apparatus.-The high-speed infra-red detection apparatus (Pyroscan) (Fig. 1) was supplied by S. Smith \& Sons (England) Ltd., and has been described in detail by Cade (1964). Briefly, the machine consists of a silvered mirror which scans the patient's abdomen horizontally and vertically. The heat image obtained is transmitted via a mirror system to an indium antimonide photoconductive cell, operating at $-200^{\circ}$ C., where it is converted into a minute electrical impulse. This signal is amplified about a million times and is then "fed" to an electrode over which electrosensitive paper passes. When the signal is "cold" a black image appears on the paper, when "hot" a white image is produced, and between the two are numerous shades of grey. A switch, incorporated into the machine after the initial series was completed, enables the 
polarity of the image-representation to be reversed, the hot image then appearing black.

Technique.-The room used for the procedure was at a temperature of approximately $20^{\circ}$ C. An environmental temperature higher than this rendered the machine incapable of detecting minor relative differences in infra-red heat emissions. With the patient in the supine position on a couch,

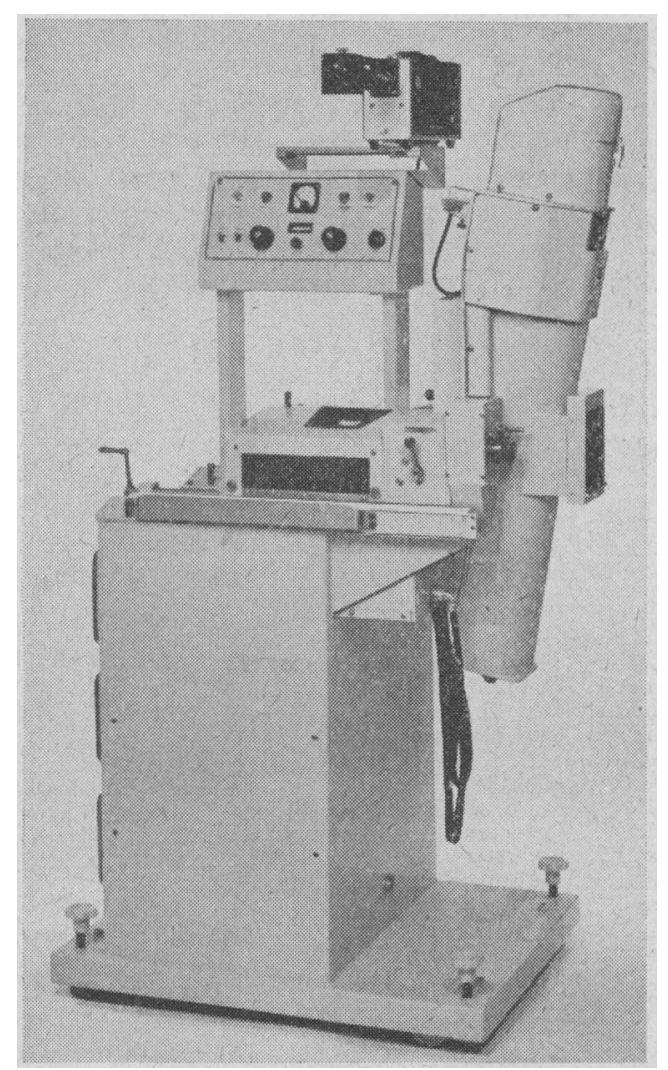

FIG. 1.-Pyroscan Mark II apparatus used in the study.

facing the "eye" of the apparatus, a plain highly silvered steel mirror at an angle of 45 degrees was placed over the abdomen at the height of the uterine fundus. This position and the mirror arrangement, different from the direct scanning methods used by previous investigators, were adopted because they enabled a view of the lower segment of the uterus to be obtained. This made more probable the detection of a placenta implanted there. It also eliminated the foreshortening effects due to a pendulous gravid abdomen. With the patient in position a cold damp towel was placed evenly over the anterior abdominal wall for about 10 minutes to reduce the surface temperature. After this interval the towel was removed, a coin placed on the umbilicus to act as a marker, and scanning begun. First, a high-speed scan was done to check the position of the patient and the focus of the apparatus. After this, slow scans were taken and the controls adjusted until a satisfactory picture was obtained.

Time of Scanning.-In the majority of cases scanning was performed at 36-40 weeks, because this was when most of the patients were admitted to hospital and were therefore available for study. In some cases of antepartum haemorrhage scanning was done soon after admission, and again later in the pregnancy.

\section{Selection of Cases}

The patients selected were divided into three groups. (1) those admitted after an antepartum haemorrhage ; confirmation of placental site was obtained in four of these cases when, at examination under anaesthesia, bleeding from placenta praevia necessitated caesarean section. (2) Those in whom the previous obstetrical history or findings during pregnancy suggested that delivery by caesarean section was likely. (3) Those, selected at random, who subsequently had normal deliveries; it was presumed, though it could not be proved, that the placental site in this group was in the body or fundus of the uterus.

Only in groups 1 and 2 could the placental site be definitely identified at the time of delivery.

\section{Difficulties Encountered}

1. High Environmental Temperature.-Initially this proved troublesome and a number of useless scans were obtained. A thermometer was placed in the room, and by opening the windows and switching on electric fans the temperature was reduced to approximately $20^{\circ} \mathrm{C}$. The quality of the scans thereafter improved considerably.

2. 50-Cycle Interference.-This is almost impossible to avoid when work must be carried out in hospital, where high-voltage radiography machines and generators are almost continuously in operation.

3. Excessive "Gain."-In an attempt to amplify a weak signal the control which adjusts the amplifier gain may be increased. This does not improve the picture but merely augments the extraneous interference present.

4. Other Faults.-These included staining and creasing of the electrosensitive paper, and were eliminated once the operator was fully conversant with the apparatus.

\section{Interpretation of Thermographs}

Before interpretation of the thermograph of a pregnant abdomen can be made one must be conversant with the thermographic pattern of the abdomen in the non-gravid state.

In the thermograph of the normal abdomen several zones of heat-emission are obvious-namely, (1) the periumbilical area -the umbilicus is invariably surrounded by a zone of increased heat-emission; (2) the inguinal areas-heat gathers in the skin folds of these regions; (3) the breasts and inframammary folds -heat also tends to gather here: pregnancy, by causing increased mammary blood-flow, further increases heat emission ; (4) the right and left subcostal regions, overlying the liver and spleen, which emit more heat than their immediate surroundings owing to their abundant vasculature.

With knowledge of this " normal " heat pattern two observers independently viewed the thermographs and attempted to identify the placental site.

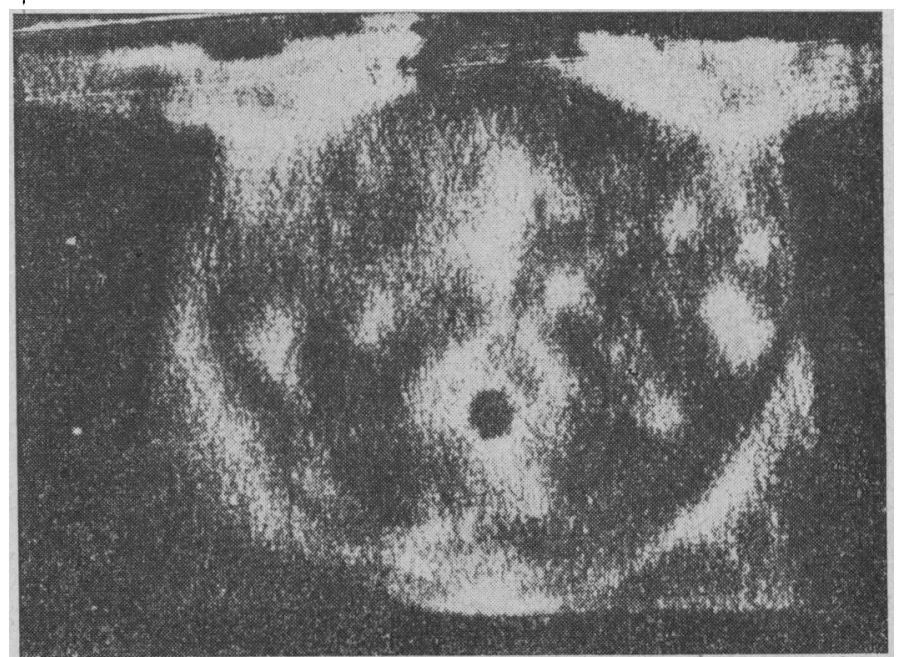

Fig. 2-Thermograph taken at 38 weeks' gestation. At caesarean section the placental site was on the upper anterior uterine wall. The patchy distribution of heat emission makes the identification of the placental site impossible. 
The groin and periumbilical hot areas present the greatest problem in interpretation. The low-lying placenta may produce a heat pattern exactly similar to the groin heat pattern. Likewise, the placenta implanted high on the anterior uterine wall may produce an area of periumbilical heat. Herein lies one of the main difficulties associated with thermography, because it is difficult to define when an area of heat emission in these regions becomes significant.

In the areas outside these zones, described above, two findings are often obtained: (1) no hot areas are seen (Fig. 4), and (2) a patchy mottling over the entire abdominal wall is encountered (Fig. 2). In both these instances location of the placental site is impossible.

After the incorporation of a switch allowing the polarity to be reversed 20 scans were done. While the image obtained was undoubtedly of better quality, the placental site could still not be identified with certainty in any case (Fig. 6).

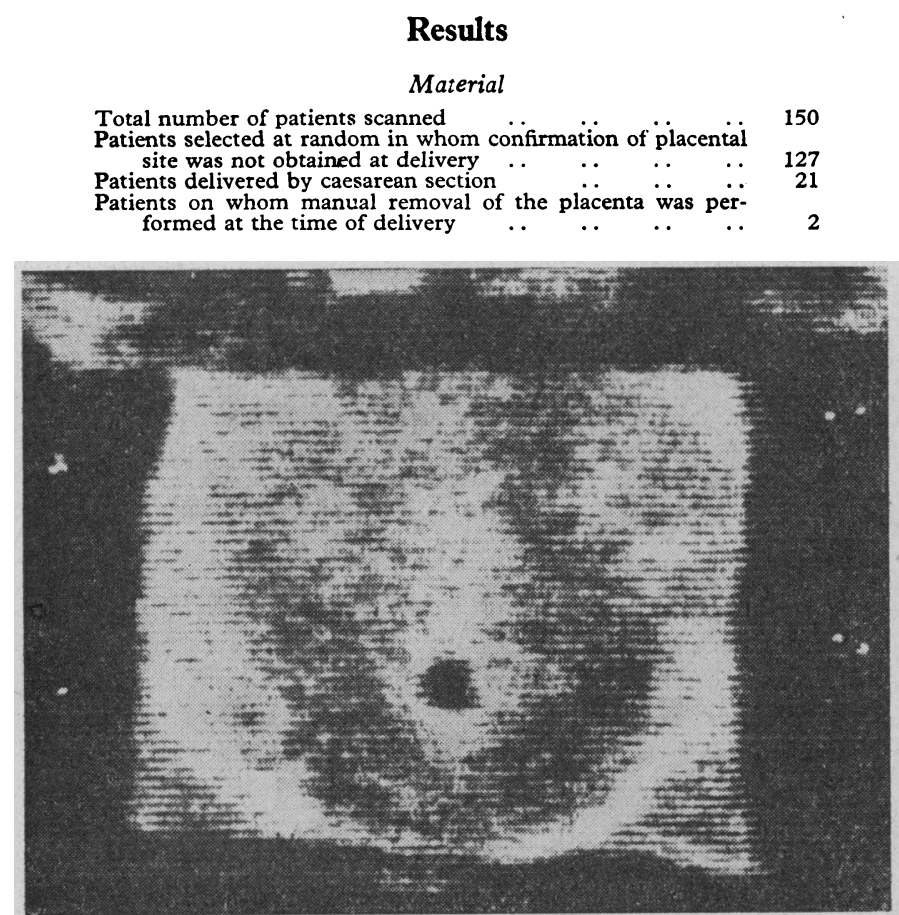

Fig. 3.-Thermograph taken at 38 weeks' gestation. Anterior type I placenta praevia found at caesarean section. The area of maximal heat emission is seen around the umbilicus and over the fundus on the right side.

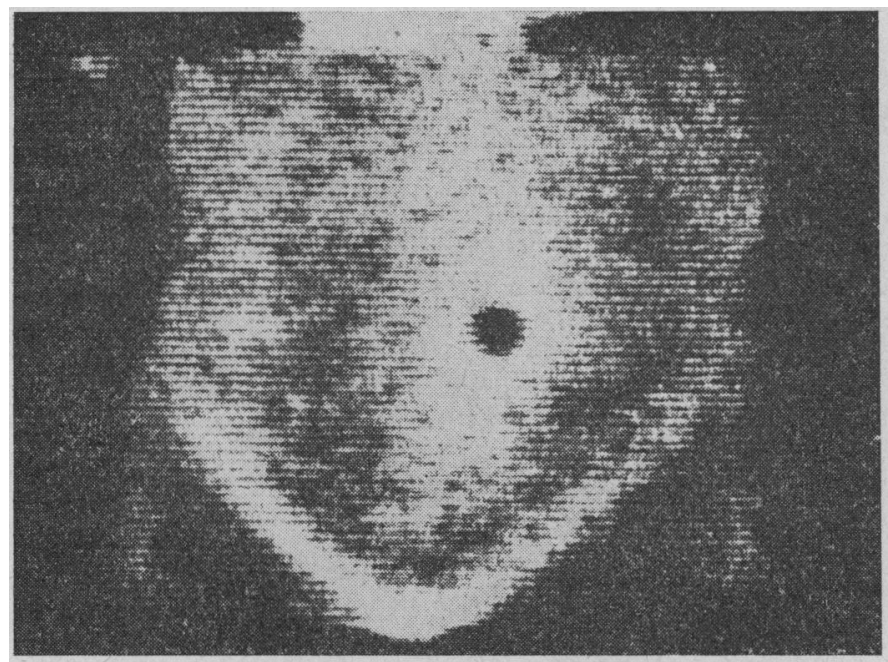

FIG. 5.- Thermograph taken at 41 weeks' gestation. The extensive area of periumbilical heat might be considered to indicate an anteriorly situated placenta. At caesarean section the placental site was high on the posterior uterine wall.
Analysis of Cases in Which Placental Site was Confirmed at Caesarean Section

Group 1-Placenta Praevia

\begin{tabular}{cccc}
\multicolumn{4}{c}{ Placental Site } \\
Posterior type II & $\ldots$ \\
Anterior & " & I & $\ldots$ \\
" & " & I & $\ldots$
\end{tabular}

Interpretation of Thermograph

Suggestive of upper anterior (Fig. 3)

No definite site seen

", ", (Fig. 4)

Group 2-Placenta in Body of Uterus

Upper posterior $(7$ cases) $\quad \ldots \quad \quad \ldots \quad$. $\quad$ No definite site seen

$$
\begin{aligned}
& \text { " } \quad " \quad \begin{array}{lllll}
(1 \text { case }) & \ldots & \ldots & \ldots & \text { Large periumbilical heat area }
\end{array}
\end{aligned}
$$

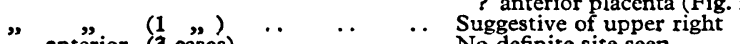

$$
\begin{aligned}
& \text { \#nterior ( }(3 \text { cases) } \quad . \quad \quad \ldots \quad \text {.. }
\end{aligned}
$$

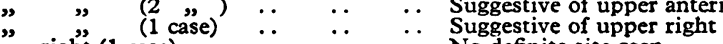

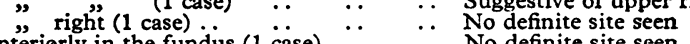

\section{Analysis of Cases in Which Placental Site was Confirmed at Manual} Removal of Placenta

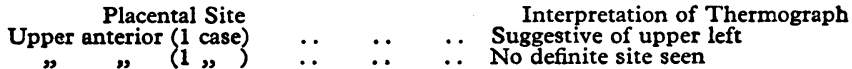

There are thus 23 patients in whom the thermographic findings have been compared with the placental site as found at the time of delivery. In 16 of these no definite placental site was demonstrated by thermography. The interpretation of the scans in the remaining seven still leaves considerable doubt in the interpreters' minds, and in no case can an unequivocal identification of the placental site be made. The

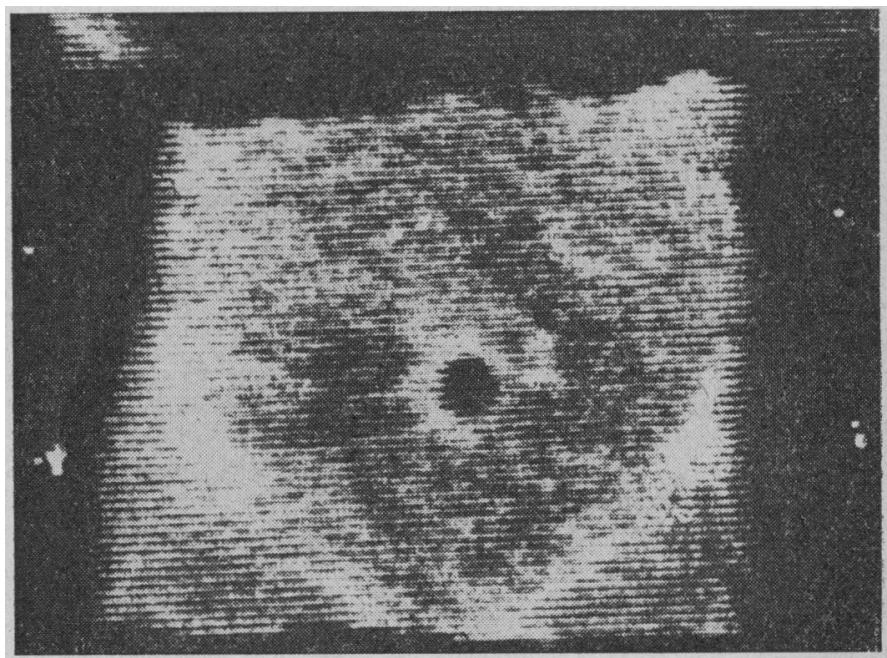

FIG. 4.-Thermograph taken at 34 weeks' gestation. Anterior type I placenta praevia found at caesarean section. No placental site can be seen.

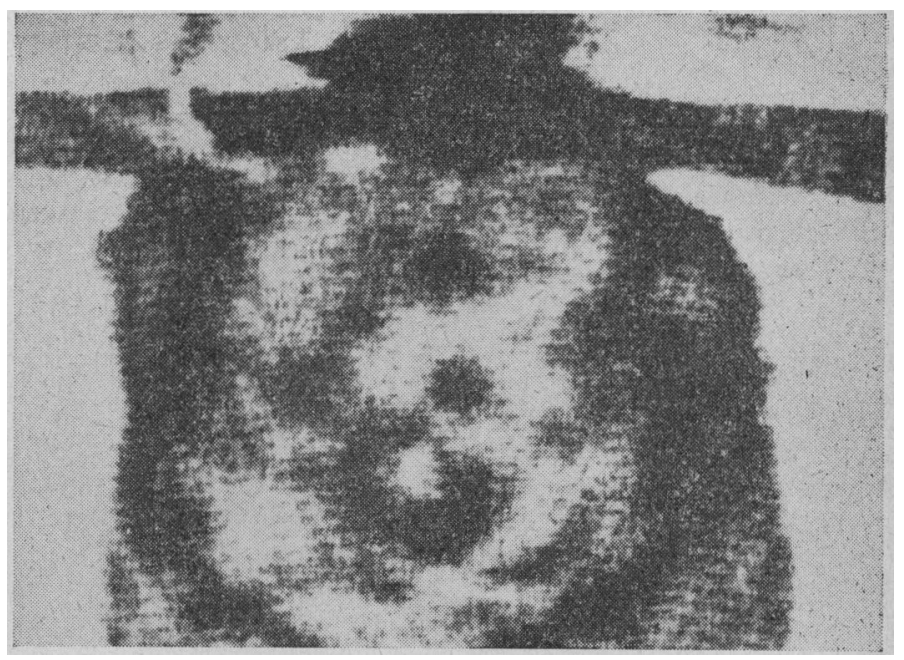

FIG. 6.-Thermograph taken at 40 weeks' gestation with the use of " reversed polarity," the hot areas appearing black. Three " hot spots" can be seen above the periumbilical hot zone. At caesarean section the
placenta was situated anteriorly in the fundus. While the "spots" may be due to the placenta, the pattern is too indefinite to make certain the identification of the placental site by thermography. 
posterior placenta-10 of which occur in the series-may produce insufficient heat emission to be detectable through the anterior abdominal wall. However, no better results are obtained in locating the anteriorly placed placenta.

Four cases of placenta praevia are included in the series. Initially, the absence, in placenta praevia cases, of an identifiable placental site on thermography was thought to be significant. However, in two of the cases subsequently demonstrated to be anterior type I placenta praevia, where the bulk of the placenta is in the body of the uterus, no site was demonstrated by thermography (Fig. 4).

Although confirmation of the placental site was not obtained, interpretation of the thermographic findings of the remaining patients, who had vaginal deliveries without third-stage complications necessitating manual removal, yielded results similar to those described above.

\section{Conclusions}

The Pyroscan is an expensive piece of apparatus that operates adequately only under ideal conditions, which are sometimes not easy to attain, and which, though not unpleasant, are not comfortable for the patient. A supply of liquid nitrogen, which requires constant replenishment, is essential in order to cool the heat-sensitive cell of the apparatus, though the latest models are now fitted with an automatic liquid-nitrogentransfer device.

Despite prolonged trial with the technical assistance of the manufacturers, it was found impossible to obtain thermographs which were of any value in determining the placental site.

An infra-red scanning apparatus, identical to the one used in this study, has been in use at the Jefferson Hospital in Philadelphia (personal communication). A lower ambient temperature $\left(18^{\circ}\right.$ C.) may have contributed to the more promising results in the series performed there.

Refinements in the apparatus may lead to an improvement in the quality of the thermographs, but until this advance is made identification of the placental site by thermography is unlikely to prove a useful obstetrical investigation.

Other applications of thermography were briefly tried while the apparatus was available. In the investigation of vascular occlusion-venous and arterial-it is of undoubted value, as it is in the investigation of breast lesions. It is considered that there is insufficient emission of heat from the placenta, even when it is situated anteriorly, to produce, on the surface of the abdominal wall, a heat pattern which the apparatus can detect.

\section{Summary}

The application of thermography in locating the placental site is investigated. The Pyroscan and its method of use are described. Of 150 patients scanned 21 were delivered by caesarean section and two had manual removal of placenta. The results show that thermography cannot be relied upon to predict the placental site accurately.

I wish to thank Professor Ian Donald for his constant help and encouragement. Also I am indebted to S. Smith \& Sons (England) Ltd. for the loan of the Pyroscan apparatus, and particularly grateful to Mr. Ashley Letts, of Smith \& Sons Ltd., for his technical advice.

\section{REFERENCES}

Barnes, R. B. (1963). Science, 140, 870.

Birnbaum, S. J., and Kliot, D. A. (1965). Obstet. and Gynec., 25, 515. Cade, C. M. (1964). Ann. N.Y. Acad. Sci., 121, 71.

Lawson, R. N. (1956). Canad. med. Ass. Ұ., 75, 309.

Lloyd Williams, K., Lloyd Williams, F., and Handley, R. S. (1960) Lancet, 2, 1378 .

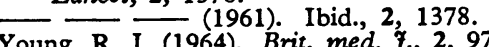

\title{
Staphylococcal Colonization of the Rectum in the Newborn
}

\author{
DINAH BARRIE,* M.B., B.S.
}

Despite the frequent isolation of Staphylococcus aureus from stool cultures of newborn infants, the finding is not always reported and its significance is uncertain. For instance, in a three-months pilot survey a moderate or heavy growth of Staph. aureus was cultured from stool specimens of 26 out of 113 infants suffering from diarrhoea, failure to thrive, and loss of weight. The present investigation was undertaken to study staphylococcal invasion of the rectum in the newborn, its relation to colonization in other sites, and its clinical significance.

\section{Materials and Methods}

Ninety-two randomly selected babies were tested on 447 occasions over the course of six months, during which time approximately 500 babies were born in the unit. The first 50 babies were examined daily from birth until discharged home, mostly on the eighth day. The remainder were examined on isolated occasions up to 7 weeks of age.

Specimens were obtained from the umbilicus, perineum, and rectum. A special device was made for taking high rectal

\footnotetext{
* Department of Clinical Microbiology, Louis Jenner Laboratories, St. Thomas's Hospital and Medical School, London.
}

swabs without contamination from the perianal skin (Fig. 1). This consisted of a piece of tissue-paper, folded over a short piece of glass tubing and secured loosely with a strip of adhesive tape. The whole was enclosed in a test-tube, sealed with cottonwool, and sterilized by autoclaving. Samples were obtained by inserting the covered end of the tube, lubricated with sterile petroleum jelly, into the rectum. With the paper sheath held in one hand the tube was advanced so that it perforated the paper and projected approximately $1 \mathrm{~cm}$. beyond the torn edges. An ordinary throat swab was then passed down the tube to sample meconium or stool. All swabs were plated direct on to salt mannite agar and incubated at $37^{\circ} \mathrm{C}$. for 48 hours. Staph. aureus was recognized by its colony appearance, mannite fermentation, and tube coagulase activity. Antibiotic sensitivity patterns were determined by the multi-disc method, and phage-typing was carried out by the method of Williams and Rippon (1952).

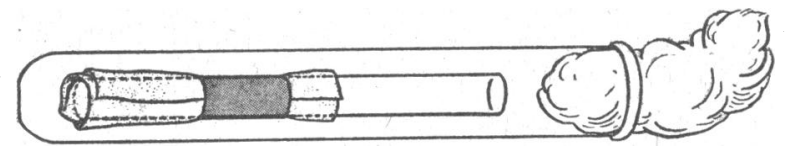

FIG. 1.-Rectal sampling device. 